\title{
Exponential Synchronization of Complex Delayed Dynamical Networks with General Topology
}

\author{
Tao Liu, Georgi M. Dimirovski, Senior Member IEEE, and Jun Zhao
}

\begin{abstract}
The global exponential synchronization of complex delayed dynamical networks possessing general topology is investigated in this contribution. The network model considered can represent both the directed and undirected weighted networks. Novel delay-dependent linear controllers are designed via Lyapunov stability theory and appropriate property of the coupling matrix. It is shown that the controlled network is globally exponentially synchronized with a given convergence rate. Two examples of typical dynamical networks with coupling delays of this class, one possesses directed and the other with undirected coupling topology, both having a Lorenz system at each node, have been used to demonstrate and verify the novel control design proposed.
\end{abstract}

\section{INTRODUCTION}

A complex network is a large set of interconnected communicating and interacting nodes where a node is a fundamental unit having specific contents and exhibiting dynamical behavior. In fact, many systems in science and technology can be modeled as complex networks, and most well known examples are: power grids, Internet, World Wide Web, metabolic systems, food webs, etc. In turn, the analysis and control of dynamical behaviors in complex networks have become a very hot topic in various disciplines [1]-[4]. Synchronization, the most important collective behavior of complex networks, has received much of the focus in recent years. A considerable number of studies on this topic have been reported [5]-[18].

Recently, [10]-[11] introduced a uniform complex network model and investigated its synchronization. [12] extended that model to the one with coupling delays, and derived both delay-independent and delay-dependent synchronization conditions. The exponential synchronization of a delayed network as well as the effects of time delays is studied in [13]. Sufficient conditions of synchronization for delayed continuous- and discrete-time network are obtained respectively in [14]. Further, in [15] and [16], the authors propose adaptive and robust adaptive, respectively, designs thereby synchronizing complex dynamical networks by control design. [17] investigated exponential synchronization of

This work was supported by the Dogus University Fund for Science and NSF of China under Grant 60574013.

Tao Liu and Jun Zhao are with Northeastern University, Key Laboratory of Integrated Automation of Process Industry, Ministry of Education, Faculty of Information Science and Engineering, Shenyang, Liaoning, 110004, P.R. of China. neuliutao@163.com; zhaojundise.neu.edu. cn

Georgi M. Dimirovski is with Dogus University, Department of Computer Engineering, Acibadem, Kadikoy, TR-34722, Istanbul, Rep. of Turkey, and with SS Cyril and Methodius University, Faculty of Electrical and Computer Engineering, Skopje, Rep. of Macedonia. gdimirovski@dogus.edu.tr a undirected controlled network with coupling delays.

It should be noted that the coupling topology is assumed to be undirected and unweighted in most of existing works. However, this simplification does not match the peculiarities of real-world networks due to the actual circumstances in far too many cases. For instance, the World Wide Web, metabolic and citation networks are all directed graphs, and some food webs, neural networks are weighted; see [18] and references therein. Another problem that deserves attention is that few works from control engineering view point, as those in [15]-[17], can be found in the literature. Sometimes the networks may not be synchronized when a controller is not added into the infrastructure of individual nodes. Thus the controlled synchronization of complex networks is believed to be a rather significant topic in both theoretical research and practical applications.

In view of these effects, our study is aimed at exponentially synchronizing delayed dynamical networks with general coupling topology via designing decentralized delaydependent linear controllers. Compared with the existing works on exponential synchronization of controlled networks, the results proposed in this paper have three distinct features: (i) the model is general; (ii) only partial information of the coupling matrix is used to design the controllers which makes the controller implementation be fairly easy in practice; (iii) the controllers designed in this paper are delay-dependent and less conservative than the exiting ones which are delay-independent.

Further this paper is written up as follows. We first introduce a more general model of delayed dynamical networks in Section II than the ones before considered in the literature. Then, in Section III, we design decentralized delaydependent linear controllers which guarantee the global exponential synchronization of the controlled network by employing the property of the coupling matrix. In Section IV, two illustrative examples for directed and undirected delayed dynamical network are given to demonstrate the effectiveness of the theoretical derivation. Conclusion and references are given thereafter.

\section{Complex Delayed Dynamical Network Model}

Consider a delayed complex dynamical network with general coupling topology which consists of $N$ nonlinearly and diffusively coupled identical nodes. Each of the nodes is an $n$-dimensional dynamical system. The state equations 
of the entire network are given as below

$$
\dot{x}_{i}=f\left(x_{i}\right)+\sum_{\substack{j=1 \\ j \neq i}}^{N} c_{i j}\left(h\left(x_{j}(t-\tau)\right)-h\left(x_{i}(t-\tau)\right)\right),
$$

where $x_{i}=\left(x_{i 1}, x_{i 2}, \ldots, x_{i n}\right)^{T} \in R^{n}, i=1,2, \ldots, N$ are state variables of node $i$, and $\tau>0$ is an arbitrary but bounded constant representing the time delay. Functions $f(\cdot) \in R^{n}$, $h(\cdot) \in R^{n}$ are assumed smooth nonlinear vector fields. Matrix $C=\left(c_{i j}\right)_{N \times N}$ is the coupling configuration matrix representing the coupling strength and topology structure of the network; if there is a connection between node $i$ and node $j(i \neq j)$, then $c_{i j}>0$; otherwise $c_{i j}=0$. The diagonal elements of matrix $C$ are defined as

$$
c_{i i}=-\sum_{\substack{j=1 \\ j \neq i}}^{N} c_{i j}
$$

Note that the network model (1) is quite general. It covers the weighted and directed network model introduced in [18]; when $C$ is symmetric, the network is weighed and undirected. On the other hand, if $h\left(x_{i}\right)=\Gamma x_{i}$ with $\Gamma=\left(r_{i j}\right)_{n \times n}$ being an inner-coupling matrix of the network, then the network model degenerates into the one of linearly and diffusively coupled network with coupling delays,

$$
\dot{x}_{i}=f\left(x_{i}\right)+\sum_{\substack{j=1 \\ j \neq i}}^{N} c_{i j} \Gamma\left(x_{j}(t-\tau)-x_{i}(t-\tau)\right)
$$

which has been discussed in the literature extensively [12][14]. Furthermore, in here the coupling matrix is not assumed to be irreducible.

Our task in this paper is to globally exponentially synchronize the states of the network (1) on the manifold defined (4) by introducing a delay-dependent linear controller into each individual node.

$$
x_{1}(t)=x_{2}(t)=\cdots=x_{N}(t)=s(t),
$$

where $s(t) \in R^{n}$ is a solution of an isolated node

$$
\dot{s}(t)=f(s(t)) .
$$

We assume that $s(t)$ is an arbitrary desired state which can be an equilibrium point, a nontrivial periodic orbit, or even a chaotic orbit.

Next, the rigorous mathematical definition of exponential synchronization for delayed dynamical networks (1) is introduced.

Definition 1. Let $X\left(t ; t_{0} ; \phi\right)=\left(x_{1}\left(t ; t_{0} ; \phi\right)^{T}, x_{2}\left(t ; t_{0} ; \phi\right)^{T}\right.$, $\left.\ldots, x_{N}\left(t ; t_{0} ; \phi\right)^{T}\right)^{T} \in R^{n N}$ be a solution of delayed dynamical network (1), where $\phi=\left(\phi_{1}^{T}, \phi_{2}^{T}, \ldots, \phi_{N}^{T}\right)^{T}, \phi_{i}=\phi_{i}(\theta) \in$ $C\left([-\tau, 0], R^{n}\right)$ are initial conditions of node $i, f: R \times \Omega \rightarrow R^{n}$, and $h: R \times \Omega \rightarrow R^{n}$ are continuously differentiable on $\Omega \subseteq R^{n}$. If there exist constants $\alpha>0, \lambda>0$ and a nonempty subset $\Lambda \subseteq \Omega$ with $\phi_{i} \in \Lambda, i=1,2, \ldots, N$ such that $X\left(t ; t_{0} ; \phi\right) \in$ $\Omega \times \cdots \times \Omega$ for all $t \geq t_{0}$, and

$$
\left\|X\left(t ; t_{0} ; \phi\right)-S\left(t ; t_{0} ; s_{0}\right)\right\| \leq \alpha \mathrm{e}^{-\lambda t} \sup _{-\tau \leq \theta \leq 0}\left\|\phi(\theta)-S_{0}\right\|
$$

$\left(\|\cdot\|\right.$ is the Euclidean norm) where $S\left(t ; t_{0} ; s_{0}\right)=$ $\left(s\left(t ; t_{0} ; s_{0}\right)^{T}, \ldots, s\left(t ; t_{0} ; s_{0}\right)^{T}\right)^{T} \in R^{n N}, \quad S_{0}=\left(s_{0}^{T}, \ldots, s_{0}^{T}\right)^{T}$, $s\left(t ; t_{0} ; s_{0}\right)$ is a solution of the system (5) with the initial condition $s_{0} \in \Omega$, then the delayed dynamical network (1) is said to realize exponential synchronization such that $\lambda$ is the exponential rate and $\Lambda \times \cdots \times \Lambda$ is called the region of synchrony of the delayed network (1).

\section{Globally Exponential Synchronization of Controlled Dynamical Network}

In this section, we study the global exponential synchronization of delayed network (1) by designing a linear controller for each node. Thus, the controlled network can be described as

$$
\dot{x}_{i}=f\left(x_{i}\right)+\sum_{\substack{j=1 \\ j \neq i}}^{N} c_{i j}\left(h\left(x_{j}(t-\tau)\right)-h\left(x_{i}(t-\tau)\right)\right)+u_{i},
$$

where $u_{i} \in R^{n}, i=1,2, \ldots, N$ are input variables of node $i$.

In order to achieve the objective on the manifold (4), we define the error vector

$$
e_{i}(t)=x_{i}(t)-s(t), \quad i=1,2, \ldots, N .
$$

Subtracting (5) from (7) yields the error dynamical system

$$
\begin{aligned}
\dot{e}_{i}(t)= & f\left(x_{i}(t)\right)-f(s(t))+\sum_{\substack{j=1 \\
j \neq i}}^{N} c_{i j}\left(g\left(e_{j}(t-\tau)\right)\right. \\
& \left.-g\left(e_{i}(t-\tau)\right)\right)+u_{i},
\end{aligned}
$$

where $g\left(e_{i}(t-\tau)\right)=h\left(x_{i}(t-\tau)\right)-h(s(t-\tau)), i=1,2, \ldots, N$. It can easily verify that $g(0)=0$. Then the global exponential synchronization problem of the dynamical network (7) is equivalent to the problem of global exponential stabilization of the error dynamical system (9), which resembles the setting in large-scale systems [19].

In the sequel, we need Assumption 1 in order to obtain our main results.

Assumption 1. There exist two positive constants $L_{1}$ and $L_{2}$, such that

$$
\begin{gathered}
\left\|f\left(x_{i}\right)-f(s)\right\| \leq L_{1}\left\|e_{i}\right\|, \\
\left\|h\left(x_{i}\right)-h(s)\right\| \leq L_{2}\left\|e_{i}\right\|,
\end{gathered}
$$

hold for $i=1,2, \ldots, N$.

Obviously, Assumption 1 is a very mild assumption and has been widely used in the literature [15]-[16].

\section{A. Synchronization of Weighted and Directed Network}

We proceed further with the following result for the case of weighted and directed network (7).

Theorem 1. Suppose Assumption 1 holds and the timeinvariant delay is $\tau \in(0, \rho]$ for some $\rho>0$. Then the delayed complex dynamical network (7) is globally exponentially synchronized for any fix time delay $\tau \in(0, \rho]$ under the set of controllers

$$
u_{i}=k_{i} e_{i}, \quad i=1,2, \ldots, N
$$


where

$$
k_{i}=-L_{1}-\lambda+c_{i i}+\frac{1}{2} \mathrm{e}^{2 \lambda \rho} L_{2}^{2}\left(c_{i}+c_{i i}\right),
$$

and $c_{i}=-\sum_{j=1, j \neq i}^{N} c_{j i}<0, c_{i i}<0, i=1,2, \ldots, N, L_{1}$ and $L_{2}$ are defined in Assumption $1, \lambda>0$ is the exponential rate available to be designed.

Proof. Select the following Lyapunov functional candidate

$$
V(t)=\mathrm{e}^{-2 \lambda \rho} \sum_{i=1}^{N} e_{i}(t)^{T} e_{i}(t)-W,
$$

where

$$
W=\sum_{i=1}^{N}\left(c_{i}+c_{i i}\right) \int_{t-\tau}^{t} \mathrm{e}^{2 \lambda(\eta-t)} g\left(e_{i}(\eta)\right)^{T} g\left(e_{i}(\eta)\right) \mathrm{d} \eta .
$$

Then the time derivative of $V(t)$ along the solution of the error system (9) is given as follows:

$$
\begin{aligned}
\dot{V}(t)= & 2 \mathrm{e}^{-2 \lambda \rho} \sum_{i=1}^{N} e_{i}(t)^{T}\left[f\left(x_{i}(t)\right)-f(s(t))+u_{i}\right. \\
& \left.+\sum_{\substack{j=1 \\
j \neq i}}^{N} c_{i j}\left(g\left(e_{j}(t-\tau)\right)-g\left(e_{i}(t-\tau)\right)\right)\right] \\
& -\sum_{i=1}^{N}\left(c_{i}+c_{i i}\right)\left\|g\left(e_{i}(t)\right)\right\|^{2}+\mathrm{e}^{-2 \lambda \tau} \sum_{i=1}^{N}\left(c_{i}+c_{i i}\right) \\
& \times\left\|g\left(e_{i}(t-\tau)\right)\right\|^{2}+2 \lambda W .
\end{aligned}
$$

Substitution the controllers (12) into previous equation, and consideration (10) in Assumption 1, yields

$$
\begin{aligned}
\dot{V}(t) \leq & 2 \mathrm{e}^{-2 \lambda \rho} \sum_{i=1}^{N}\left(-\lambda+c_{i i}\right)\left\|e_{i}(t)\right\|^{2}+2 \mathrm{e}^{-2 \lambda \rho} \sum_{i=1}^{N} \sum_{\substack{j=1 \\
j \neq i}}^{N} c_{i j} e_{i}^{T} \\
& \times\left(g \left(e_{j}(t-\tau)-g\left(e_{i}(t-\tau)\right)+\sum_{i=1}^{N}\left(c_{i}+c_{i i}\right)\left(L_{2}^{2}\left\|e_{i}(t)\right\|^{2}\right.\right.\right. \\
& \left.-\left\|g\left(e_{i}(t)\right)\right\|^{2}\right)+\mathrm{e}^{-2 \lambda \tau} \sum_{i=1}^{N}\left(c_{i}+c_{i i}\right)\left\|g\left(e_{i}(t-\tau)\right)\right\|^{2} \\
& +2 \lambda W
\end{aligned}
$$

From (11) in Assumption 1, we have $\left\|g\left(e_{i}\right)\right\|=\| h\left(x_{i}\right)-$ $h(s)\left\|\leq L_{2}\right\| x_{i}-s\left\|=L_{2}\right\| e_{i} \|$, and this is to say

$$
\sum_{i=1}^{N}\left(c_{i}+c_{i i}\right) L_{2}^{2}\left\|e_{i}(t)\right\|^{2} \leq \sum_{i=1}^{N}\left(c_{i}+c_{i i}\right)\left\|g\left(e_{i}(t)\right)\right\|^{2} .
$$

On the other hand, $\mathrm{e}^{-2 \lambda \tau} \geq \mathrm{e}^{-2 \lambda \rho}$, where $c_{i}<0, c_{i i}<0$, $0<\tau \leq \rho$, hence we have

$$
\begin{aligned}
\dot{V}(t) \leq & 2 \mathrm{e}^{-2 \lambda \rho} \sum_{i=1}^{N}\left(-\lambda+c_{i i}\right)\left\|e_{i}(t)\right\|^{2}+2 \mathrm{e}^{-2 \lambda \rho} \sum_{i=1}^{N} \sum_{\substack{j=1 \\
j \neq i}}^{N} c_{i j} e_{i}^{T} \\
& \times\left(g \left(e_{j}(t-\tau)-g\left(e_{i}(t-\tau)\right)+\mathrm{e}^{-2 \lambda \rho} \sum_{i=1}^{N}\left(c_{i}+c_{i i}\right)\right.\right. \\
& \times\left\|g\left(e_{i}(t-\tau)\right)\right\|^{2}+2 \lambda W .
\end{aligned}
$$

From section II apparently we have

$$
\sum_{i=1}^{N} \sum_{\substack{j=1 \\ j \neq i}}^{N} c_{j i}\left\|g\left(e_{i}(t-\tau)\right)\right\|^{2}=\sum_{i=1}^{N} \sum_{\substack{j=1 \\ j \neq i}}^{N} c_{i j}\left\|g\left(e_{j}(t-\tau)\right)\right\|^{2} .
$$

Due to property (2), (14) and with $c_{i}=-\sum_{j=1, j \neq i}^{N} c_{j i}$, one obtains

$$
\begin{aligned}
\dot{V}(t) \leq & -2 \lambda \mathrm{e}^{-2 \lambda \rho} \sum_{i=1}^{N}\left\|e_{i}(t)\right\|^{2}-2 \mathrm{e}^{-2 \lambda \rho} \sum_{\substack { i=1 \\
\begin{subarray}{c}{j \neq 1 \\
j \neq i{ i = 1 \\
\begin{subarray} { c } { j \neq 1 \\
j \neq i } }\end{subarray}}^{N} c_{i j}\left\|e_{i}(t)\right\|^{2} \\
& +2 \mathrm{e}^{-2 \lambda \rho} \sum_{i=1}^{N} \sum_{\substack{j=1 \\
j \neq i}}^{N} c_{i j} e_{i}(t)^{T}\left(g\left(e_{j}(t-\tau)\right)-g\left(e_{i}(t-\tau)\right)\right) \\
& -\mathrm{e}^{-2 \lambda \rho} \sum_{i=1}^{N} \sum_{\substack{j=1 \\
j \neq i}}^{N} c_{i j}\left(\left\|g\left(e_{j}(t-\tau)\right)\right\|^{2}+\left\|g\left(e_{i}(t-\tau)\right)\right\|^{2}\right) \\
& +2 \lambda W \quad 2 \lambda W-2 \lambda \mathrm{e}^{-2 \lambda \rho} \sum_{i=1}^{N}\left\|e_{i}(t)\right\|^{2} \\
= & -\mathrm{e}^{-2 \lambda \rho} \sum_{i=1}^{N} \sum_{\substack{j=1 \\
j \neq i}}^{N} c_{i j}\left\|e_{i}(t)-g\left(e_{j}(t-\tau)\right)\right\|^{2} \\
& -\mathrm{e}^{-2 \lambda \rho} \sum_{\substack{i=1 \\
j}}^{N} \sum_{\substack{j=1 \\
j \neq i}}^{N} c_{i j}\left\|e_{i}(t)+g\left(e_{i}(t-\tau)\right)\right\|^{2} .
\end{aligned}
$$

Now it is readily seen that

$$
\begin{aligned}
& -\sum_{i=1}^{N} \sum_{\substack{j=1 \\
j \neq i}}^{N} c_{i j}\left\|e_{i}(t)-g\left(e_{j}(t-\tau)\right)\right\|^{2} \\
& -\sum_{i=1}^{N} \sum_{\substack{j=1 \\
j \neq i}}^{N} c_{i j}\left\|e_{i}(t)+g\left(e_{i}(t-\tau)\right)\right\|^{2} \leq 0
\end{aligned}
$$

where $c_{i j} \geq 0$, for $i, j=1,2, \ldots, N, i \neq j$. Therefore, it follows that

$$
\dot{V}(t) \leq-2 \lambda V(t) .
$$

By calculating integration on both sides of inequality (15), we get

$$
V(t) \leq \mathrm{e}^{-2 \lambda\left(t-t_{0}\right)} V\left(t_{0}\right) .
$$

Further, with regard to (13), it is readily inferred

$$
a\|e(t)\|^{2} \leq V(t) \leq b\left\|e_{t_{0}}\right\|^{2},
$$

where $e=\left(e_{1}^{T}, e_{2}^{T}, \ldots, e_{N}^{T}\right)^{T},\left\|e_{t_{0}}\right\|=\sup _{-\tau \leq \theta \leq 0}\left\|e\left(t_{0}+\theta\right)\right\|$, $a=\mathrm{e}^{-2 \lambda \rho}, b=\mathrm{e}^{-2 \lambda \rho}+c, c=\max \left\{\left|c_{1}\right|, \ldots,\left|c_{N}\right|,\left|c_{11}\right|, \ldots\right.$, $\left.\left|c_{N N}\right|\right\}$. By making use of (16) and (17), finally we obtain:

$$
\|e(t)\| \leq \sqrt{\frac{b}{a}} \mathrm{e}^{-\lambda\left(t-t_{0}\right)}\left\|e_{t_{0}}\right\| .
$$

Therefore, in closed loop under the controllers (12), it follows that the error dynamical system (9) is globally exponentially stable at the equilibrium set $e_{i}=0, i=1,2, \ldots, N$ 
with the exponential rate $\lambda$. Consequently, the synchronous solution of delayed dynamical network (7) is globally exponentially stable. Thus, the proof is completed.

In a special case when $h\left(x_{i}\right)=\Gamma x_{i}$, i.e., the network is linearly coupled, the complex network model (1) degenerates into (3). Also, note that the inequality $\left\|\Gamma e_{i}\right\| \leq\|\Gamma\| \cdot\left\|e_{i}\right\|$ holds. This way, for the network (3) we obtain the next corollary.

Corollary 1. Consider the complex network (3) with the time-invariant delay $\tau \in(0, \rho]$. If the inequality (10) in Assumption 1 holds, then the synchronous solution of complex dynamical network (3) is globally exponentially stable under controllers

$$
u_{i}=k_{i} e_{i}, \quad i=1,2, \ldots, N
$$

where

$$
k_{i}=-L_{1}-\lambda+c_{i i}+\frac{1}{2} \mathrm{e}^{2 \lambda \rho}\left(c_{i}+c_{i i}\right)\|\Gamma\|^{2},
$$

and $c_{i}, i=1,2, \ldots, N$, and $L_{1}$ are defined as in Theorem 1 .

Until now, we have investigated the synchronization of weighted and directed network.

\section{B. Synchronization of Weighted and Undirected Network}

Some real-world networks have undirected graph topology structure. In this case, it appears the form of the controllers proposed in Theorem 1 will be simplified. When the network is undirected, i.e. $C$ is a symmetric matrix, then we have

$$
c_{i i}=-\sum_{\substack{j=1 \\ j \neq i}}^{N} c_{i j}=-\sum_{\substack{j=1 \\ j \neq i}}^{N} c_{j i}
$$

and

$$
\sum_{i=1}^{N} \sum_{\substack{j=1 \\ j \neq i}}^{N} c_{i j}\left\|g\left(e_{i}(t)\right)\right\|^{2}=\sum_{i=1}^{N} \sum_{\substack{j=1 \\ j \neq i}}^{N} c_{i j}\left\|g\left(e_{j}(t)\right)\right\|^{2} .
$$

It is therefore that the controllers reduce to a more simple form. This is addressed in Theorem 2.

Theorem 2. Suppose Assumption 1 holds and the timeinvariant delay is $\tau \in(0, \rho]$ for some $\rho>0$. Then the delayed complex dynamical network (7) with weighted and undirected topology is globally exponentially synchronized for any fix time delay $\tau \in(0, \rho]$ under controllers

$$
u_{i}=k_{i} e_{i}, \quad i=1,2, \ldots, N
$$

where

$$
k_{i}=-L_{1}-\lambda+\left(1+\mathrm{e}^{2 \lambda \rho} L_{2}^{2}\right) c_{i i},
$$

and $L_{1}, L_{2}$ are defined as in Theorem 1 .

Proof. Select the following Lyapunov functional candidate

$$
\begin{aligned}
V(t)= & \mathrm{e}^{-2 \lambda \rho} \sum_{i=1}^{N} e_{i}^{T} e_{i}-2 \sum_{i=1}^{N} c_{i i} \int_{t-\tau}^{t} \mathrm{e}^{2 \lambda(\eta-t)} g\left(e_{i}(\eta)\right)^{T} \\
& \times g\left(e_{i}(\eta)\right) \mathrm{d} \eta .
\end{aligned}
$$

By using the property (19) and (20), one can get that the time derivative of $V(t)$ along the error system (9) satisfies the inequality

$$
\dot{V}(t) \leq-2 \lambda V(t) .
$$

Further the procedure of the proof is rather similar to that of Theorem 1, and it is omitted.

Remark 1. In reality, it is often quite difficult to get the entire information on the coupling matrix $C$. To overcome this constraint, in this paper, we use partial information of the matrix $C$ to design the controllers. For the directed network, the diagonal elements $c_{i i}$ and the negative column sum of offdiagonal elements $c_{i}$ are used to synthesize the decentralized controllers. In particular, only the diagonal elements $c_{i i}$ are needed for the designed controllers to synchronize the undirected network. These make fairly easy the controllers implementation in practice.

Remark 2. The controller design method proposed in this section can be applied to some uncertain delayed network. When the exact value of $c_{i}$ and $c_{i i}$ are unavailable, their lower bounds $\tilde{c}_{i}$ and $\tilde{c}_{i i}$ can be estimated instead. Then these lower bounds should replace parameters $c_{i}$ and $c_{i i}$ in the designed controllers. Thus adjusted controllers still exponentially synchronize the corresponding uncertain network.

Remark 3. For the delay parameter, it is very hard to get exact information of $\tau$, but there are still methods to give an upper bound $\rho$ on $\tau$ to synthesize the delay-dependent controllers. Further, with some slight change, the controllers can also be applied to the network with time-varying coupling delays $0<\tau(t) \leq \rho$ where the delay derivative is less than 1.

Remark 4. It should be noted that our results are less conservative than the ones proposed in [17]. The model considered in this paper is quite general. The nodes are coupled nonlinearly, and coupling topology can represent both directed and undirected weighted network. Moreover, our controllers are delay-dependent whereas in [17] the controllers are delay-independent, which makes them conservative especially in cases of networks with large coupling delays.

\section{ILlustrative Examples}

To show the effectiveness of proposed synchronization criteria, we consider a delayed dynamical network with nonlinearly coupled Lorenz systems. It is known that the solution of the trajectory of the Lorenz system approaches a chaotic attractor, and a single Lorenz system is described by

$$
\left(\begin{array}{c}
\dot{x}_{1} \\
\dot{x}_{2} \\
\dot{x}_{3}
\end{array}\right)=A\left(\begin{array}{l}
x_{1} \\
x_{2} \\
x_{3}
\end{array}\right)+\left(\begin{array}{c}
0 \\
-x_{1} x_{3} \\
x_{1} x_{2}
\end{array}\right),
$$

where

$$
A=\left(\begin{array}{ccc}
-p_{1} & p_{1} & 0 \\
p_{3} & -1 & 0 \\
0 & 0 & -p_{2}
\end{array}\right)
$$


$p_{1}=10, p_{2}=8 / 3, p_{3}=28$. And the networked system is defined as:

$$
\begin{aligned}
\left(\begin{array}{c}
\dot{x}_{i 1} \\
\dot{x}_{i 2} \\
\dot{x}_{i 3}
\end{array}\right)= & A\left(\begin{array}{c}
x_{i 1} \\
x_{i 2} \\
x_{i 3}
\end{array}\right)+\left(\begin{array}{c}
0 \\
-x_{i 1} x_{i 3} \\
x_{i 1} x_{i 2}
\end{array}\right) \\
& +\sum_{\substack{j=1 \\
j \neq i}}^{N} c_{i j}\left(h\left(x_{j}(t-\tau)\right)-h\left(x_{i}(t-\tau)\right)\right)+u_{i},
\end{aligned}
$$

where $h\left(x_{i}(t-\tau)\right)=\left(p_{1}\left(x_{i 2}(t-\tau)-x_{i 1}(t-\tau)\right), 0, x_{i 1}(t-\right.$ $\left.\tau) x_{i 2}(t-\tau)-p_{2} x_{i 3}(t-\tau)\right)^{T}$, and $\tau=0.1$.

Similar to [15], Assumption 1 holds. We simulate the above delayed network with two different coupling topology structures: (a) directed network; (b) undirected network. The details of the simulation experiments are presented in the following two subsections.

\section{A. Directed Network}

Here, we consider the directed network with 10 dynamical nodes. Select the asymmetric coupling matrix as

$$
C=\left(\begin{array}{ccccc}
-0.1 & 0 & 0 & 0 & 0.1 \\
0 & -0.3 & 0 & 0.1 & 0 \\
0 & 0 & -0.5 & 0.1 & 0.1 \\
0.1 & 0.1 & 0.1 & -0.8 & 0.1 \\
0 & 0 & 0.1 & 0.1 & -0.3 \\
0 & 0.1 & 0.1 & 0.1 & 0 \\
0 & 0.1 & 0 & 0 & 0 \\
0 & 0 & 0 & 0.1 & 0.1 \\
0 & 0 & 0 & 0 & 0 \\
0 & 0.1 & 0 & 0 & 0 \\
& & & & \\
0 & 0 & 0 & 0 & 0 \\
0 & 0.1 & 0 & 0 & 0.1 \\
0.1 & 0.1 & 0 & 0 & 0.1 \\
0.1 & 0 & 0.1 & 0.1 & 0.1 \\
0 & 0 & 0.1 & 0 & 0 \\
-0.6 & 0.1 & 0.1 & 0.1 & 0 \\
0.1 & -0.3 & 0 & 0.1 & 0 \\
0 & 0 & -0.2 & 0 & 0 \\
0 & 0.1 & 0 & -0.1 & 0 \\
0 & 0 & 0 & 0 & -0.1
\end{array}\right) .
$$

According to Theorem 1 in Section III, one can synchronize the network (23) by design of the following linear controllers

$$
u_{i}=k_{i} e_{i}, \quad i=1,2, \ldots, N
$$

where $\lambda=1, \rho=\tau=0.1, s(0)=(4,5,6)^{T}$.

Fig.1-Fig.3 show the synchronous errors $e_{i 1}, e_{i 2}, e_{i 3}, i=$ $1,2, \ldots, 10$ of the network (23) with directed graph topology. Apparently, all the synchronization errors do globally exponentially converge to zero with the given exponential rate $\lambda=1$, i.e., the synchronous solution is exponentially stable for delayed dynamical network (23)-(24).

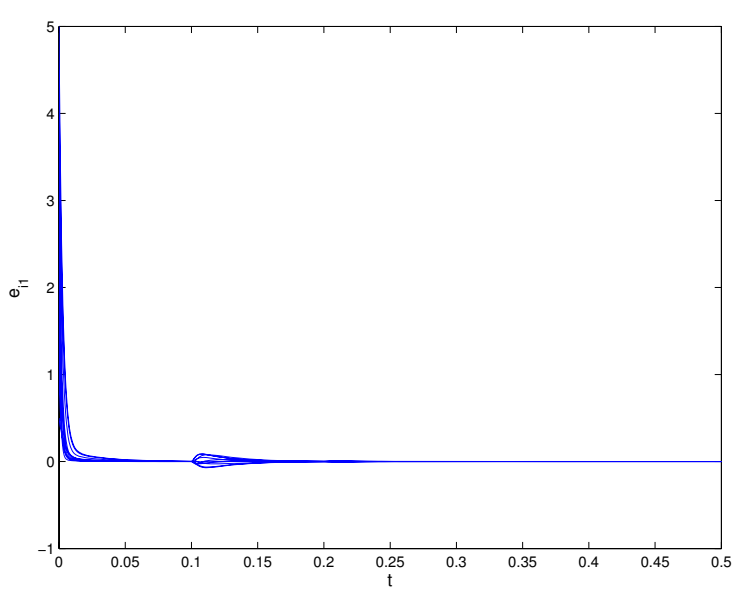

Fig. 1. Synchronization errors $e_{i 1}$ of the directed network.

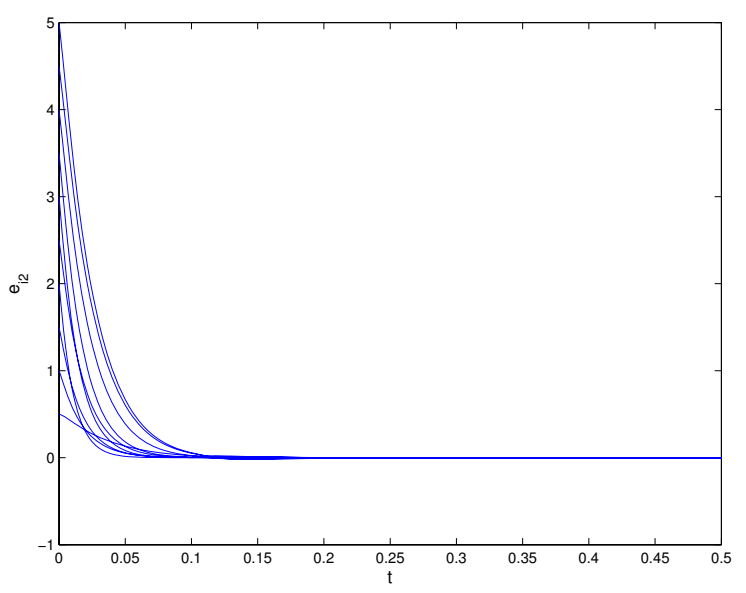

Fig. 2. Synchronization errors $e_{i 2}$ of the directed network.

\section{B. Undirected Network}

Consider the 50-node delayed network (23) generated by B-A scale-free model where the coupling matrix $C$ is symmetric in this case. Based on Theorem 2 in Section III, the linear controllers (21) exponentially synchronize the network (23), where $\lambda=1, \rho=\tau=0.1, s(0)=(4,5,6)^{T}$. The simulation results are shown in Fig.4-Fig.6, respectively, where one can get that all the states of the undirected network indeed exponentially synchronize to that of isolated node (5).

\section{CONCLUSIONS}

The problem of global exponential synchronization for complex networks with nonlinear dynamical nodes and coupling delays has been investigated in this paper. The complex network with decentralized controllers is considered as an integrally large-scale nonlinear system with a special structure. An adequate Lyapunov functional is constructed to deal with the problem of controlled synchronization as to ensure the closed exponential stability. Several network synchronization criteria for such a network have been obtained. With the topology of the network, decentralized delay-dependent linear controllers are designed such that the 


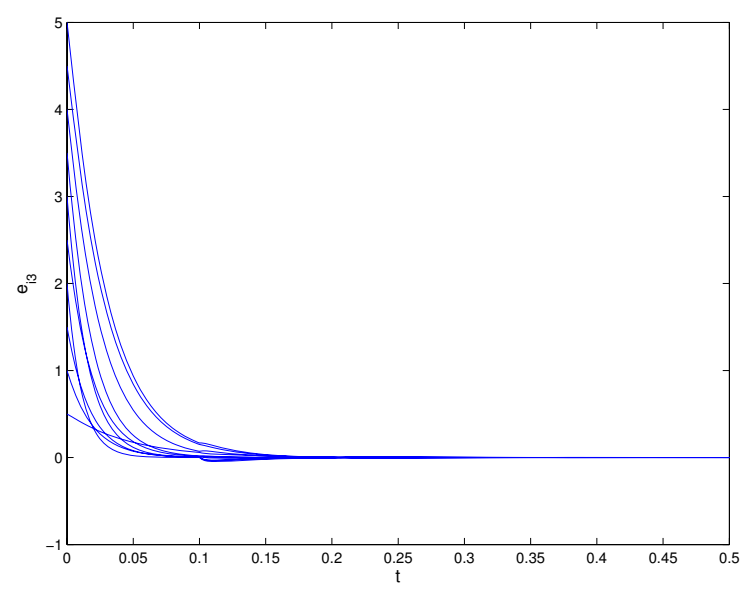

Fig. 3. Synchronization errors $e_{i 3}$ of the directed network.

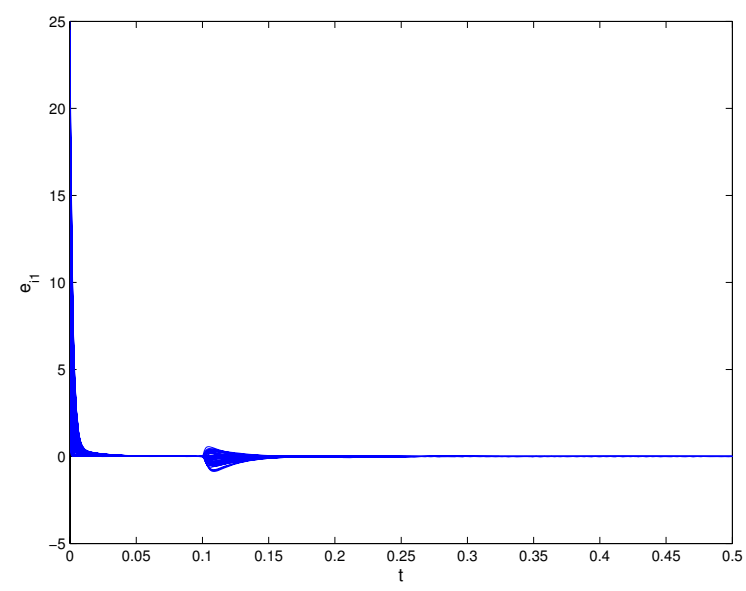

Fig. 4. Synchronization errors $e_{i 1}$ of the undirected scale-free network.

global exponential synchronization for the delayed networks is solved. Two numerical examples of delayed networks with different coupling topology are given, which demonstrate the effectiveness of proposed results.

\section{REFERENCES}

[1] S.H. Strogatz, Exploring complex networks, Nature, vol. 410, 2001, pp. 268-276.

[2] R. Albert and A.L. Barabási, Statistical mechanics of complex networks, Rev. Mod. Phys., vol. 74, 2002, pp 47-91.

[3] M. Newman, The structure and functions of complex networks, SIAM Rev., vol. 45, 2003, pp 167-256.

[4] S. Boccalettia, V. Latora, Y. Moreno, M. Chavezf and D.U. Hwang, Complex networks: structure and dynamics, Phys. Reports, vol. 424, 2006, pp 175-308.

[5] M.Z. Ding and Y.M. Yang, Stability of synchronous chaos and on-off intermittency in coupled map lattices, Phys. Rev. E, vol. 56(4),1997, pp 4009-4016.

[6] C.W. Wu and L.O. Chua, Synchronization in an array of linearly coupled dynamical systems, IEEE Trans. Circuits Syst. I, vol. 42, 1995, pp. 430-447.

[7] H. Hong, M.Y. Choi and B.J. Kim, Synchronization on small-world networks, Phys. Rev. E, vol. 65, 2002, p 026139.

[8] M. Barahona and L.M. Pecora, Synchronization in small-world systems, Phys. Rev. Lett., vol. 89, 2002, p 054101.

[9] J. Lü and G. Chen, A time-varying complex dynamical network model and its controlled synchronization criteria, IEEE Trans Auto. contr., vol. 50, 2005, pp 841-846.

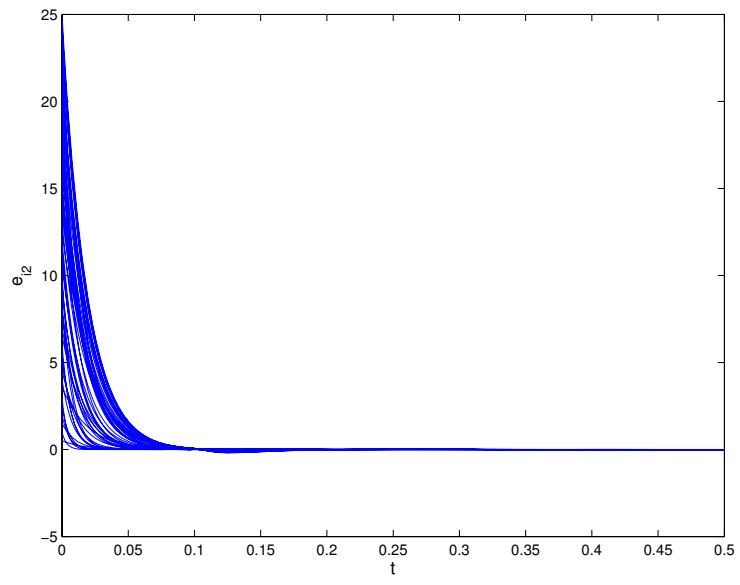

Fig. 5. Synchronization errors $e_{i 2}$ of the undirected scale-free network.

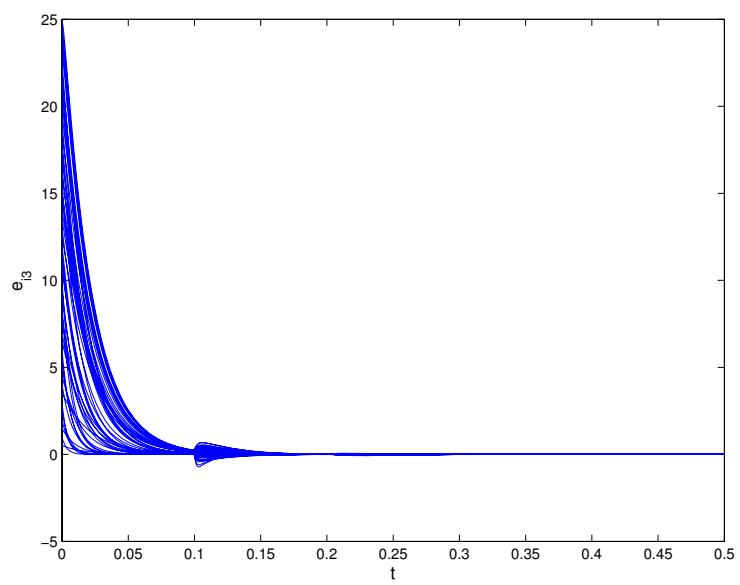

Fig. 6. Synchronization errors $e_{i 3}$ of the undirected scale-free network.

[10] X.F. Wang and G. Chen, Synchronization in small-word dynamical networks, Int. J. Bifurcation Chaos, vol. 12, 2002, pp 187-192.

[11] X.F. Wang and G. Chen, Synchronization in scale-free dynamical networks: robustness and fragility, IEEE Trans. Circuits Syst. I, vol. 49, 2002, pp 54-62.

[12] C. Li and G. Chen, Synchronization in general complex dynamical networks with coupling delays, Phys. A, vol. 343, 2004, pp 263-278.

[13] J. Zhou and T. Chen, Synchronization in general complex delayed dynamical networks, IEEE Trans. Circuits Syst. I, vol. 53(3), 2006 , pp 733-744.

[14] H. Gao, J. Lam and G. Chen, New criteria for synchronization stability of general complex dynamical networks with coupling delays, Phys. Lett. A, doi:10.1016/j.physleta.2006.08.033.

[15] J. Zhou, J. Lu and J. Lü, Adaptive synchronization of an uncertain complex dynamical network, IEEE Trans Auto. Contr., vol. 51, 2006, pp 652-656.

[16] Z. $\mathrm{Li}$ and G. Chen, Robust adaptive synchronization of uncertain dynamical networks, Phys. Lett. A, vol. 324, 2004, pp 166-178.

[17] Z. Li, G. Feng and D. Hill, Controlling complex dynamical networks with coupling delays to a desired orbit, Phys. Lett. A, vol.359, 2006, pp 42-46

[18] L.Y. Xiang, Z.X. Liu, Z.Q. Chen, F. Chen and Z.Z. Yuan, Pinning control of complex dynamical networks with general topology, Phys. A, doi:10.1016/j.physa.2006.12.037.

[19] X.-Z. Liu, G. M. Dimirovski, Y.-W. Jing, and S.-Y. Zhang, Robust decentralized stabilization for large-scale systems with multiple timevarying delays and nonlinear uncertainties, in Proceedings of the IEEE CSS Conference on Control Applications, Istanbul, TR, June 23-25. The IEEE, Piscataway, NJ, vol. 1, 2003, pp. 296-300. 